\title{
Protocol Adherence in the Intensive Care Unit for the Management of Adult Patients Admitted with Acute Aneurysmal Subarachnoid Hemorrhage
}

\author{
Lily Taylor $^{1}$ Andrew S. Lane ${ }^{2,3}$ \\ ${ }^{1}$ Department of Internal Medicine, Orange Health Service, Orange \\ NSW, Australia \\ ${ }^{2}$ Sydney Medical School, University of Sydney, Australia \\ ${ }^{3}$ Discipline of Intensive Care Medicine, Nepean Hospital, \\ Sydney, Australia
}

J Neuroanaesthesiol Crit Care 2021;8:99-105.
Address for correspondence Andrew S. Lane, MBBS, PhD, FCICM, MQHR, Nepean Clinical School, Derby Street, Penrith, Orange NSW 2773, Australia (e-mail: Stuart.lane@sydney.edu.au).

\begin{abstract}
Background There are recognized protocols that exist for management with minimal data regarding protocol adherence. We conducted a retrospective analysis of aneurysmal subarachnoid hemorrhage $(\mathrm{aSAH})$ to determine whether the level of protocol adherence varied based on patient demographics or specific aspects of management.

Materials and Methods All cases of aSAH admitted to a tertiary-level intensive care unit (ICU) from 2014 to 2016 were identified from the Australia and New Zealand Intensive Care Society Core Database as well as the clinical records system. ICU demographic and descriptive data for protocol adherence, were collected from admission to discharge up to 22 days, or until death whichever was earlier.

Results A total of 58 cases of aSAH were registered; mean age was 56.7 years, $70.7 \%$ of patients were female, and mean length of stay was 12.6 days. World Federation of Neurosurgical Societies (WFNS) scale was documented more than Fisher grading. Of the 58 cases, $63.7 \%$ (37) underwent surgical clipping, with 83.7\% (30) patients having this surgery within 48 hours. SBP/MAP were the most consistently recorded observations within protocol ranges, with adherence of $82.4 \%$ and $82.1 \%$, respectively. Thirty-two percent of temperature measurements were outside of the normothermic range of 36.5 to $37.5^{\circ} \mathrm{C}$ with a mean adherence of $47.5 \%$ (standard deviation $= \pm 0.24$, median $=40$ ). There was no correlation between adherence and patient, disease, or admission factors.

Keywords

- acute aneurysmal subarachnoid hemorrhage

- blood pressure control

- protocol adherence

Conclusion This study demonstrated that there was no association between variation in protocol adherence based on age, admission dates, or disease factors including WFNS grade and Fisher scale. Best protocol adherence protocol for the management of aSAH within the ICU was blood pressure control. Areas for improvement were documentation of the WFNS and Fisher grading, and temperature measurement and management.
\end{abstract}

DOI https://doi.org/ $10.1055 / \mathrm{s}-0040-1718504$ ISSN 2348-0548. (c) 2021. Indian Society of Neuroanaesthesiology and Critical Care. This is an open access article published by Thieme under the terms of the Creative Commons Attribution-NonDerivative-NonCommercial-License, permitting copying and reproduction so long as the original work is given appropriate credit. Contents may not be used for commercial purposes, or adapted, remixed, transformed or built upon. (https://creativecommons.org/licenses/by-nc-nd/4.0/).

Thieme Medical and Scientific Publishers Pvt. Ltd. A-12, 2nd Floor, Sector 2, Noida-201301 UP, India 


\section{Introduction}

Aneurysmal subarachnoid hemorrhage (aSAH) is a worldwide health burden with permanent disability rates and a high mortality. ${ }^{1-3}$ The overall prognosis post-aSAH is poor. ${ }^{4,5}$ Survival is dependent on multiple factors, including the severity of the initial bleeding, vasospasm, and presence of delayed cerebral ischemia (DCI). ${ }^{6,7}$

Due to the multifactorial nature of this injury, many studies exist exploring optimal approach to management. Consensus supports the strict management of blood pressure, intracranial pressure, correction of coagulopathy, and nimodipine administration for vasospasm prophylaxis. ${ }^{8,9}$ However, controversies exist regarding seizure prophylaxis ${ }^{10,11}$ and antifibrinolytic use. ${ }^{12}$ In addition, aneurysms can be either angiographically coiled or surgically clipped following aSAH. Major studies including the Barrow Ruptured Aneurysmal Trial $)^{13}$ and International Subarachnoid Aneurysm Trial ${ }^{14}$ trials found that endovascular coiling provides a better outcome in terms of survival free of disability at 1 -year post-aSAH. ${ }^{15,16}$

It is a challenge for clinicians and clinical staff to process the quantity and validity of available clinical and scientific information regarding complex management of aSAH. Once a best-practice model of treatment is decided upon, which is often not without controversy, it becomes difficult to monitor the management of aSAH accurately and consistently according to guidelines.

The use of validated protocols in everyday practice provides an opportunity for objective, evidence-based treatment regimens to be employed. ${ }^{17-19}$ Despite established evidence, junior staff face barriers regarding practice implementation, with the decision-making focus often shifting from best patient practice to senior medical staff preference. To avoid this variation in management within our tertiary-referral intensive care unit (ICU), the management of aSAH is governed by a documented protocol: "Management of Aneurysmal aSAH in Adults" (see -Supplementary Material, available in the online version).

For protocols to remain up to date and effective, it is necessary to provide periodic feedback regarding compliance and investigate barriers to adherence. ${ }^{20,21}$ The current aSAH protocol is reviewed annually according to what is the best practice $^{8}$; however, adherence has not been assessed among the larger patient population within our ICU.

This study aimed to assess adherence to the current protocol for the management of aSAH, and to determine if there was an association with level of protocol adherence based on patient factors (age, sex), department factors (day of admission or discharge), disease factors (Fisher grade, World Federation of Neurosurgical Societies [WFNS] score), or specific aspects of management (e.g., blood pressure control and temperature control).

\section{Methods}

\section{Study Design}

We performed a retrospective analysis from the medical record data of patients diagnosed with aSAH admitted to a tertiary-referral ICU in Australia from January 2014 to
December 2016. This study was approved by the Low and Negligible Risk (LNR) Human Research Ethics Committee of the Nepean Blue Mountains Local Health Districts (Reference Number 1755A). Patient consent was not required due to the retrospective nature of the study. Data was obtained from the Electronic Medical Records (EMR) system, unique to the ICU. This database contains information regarding the patient identifiers, diagnosis, management, and follow-up from admission to discharge or death.

This study specifically looked at management and adherence to the current Nepean aSAH protocol from admission to discharge before 22 days, admission up to 22 days, or admission to death. A cutoff of 22 days was chosen, as this is when the protocolized management ends. This day was originally chosen for the protocol, to align with the completion of 21 days nimodipine for the prevention of vasospasm.

The following binary data points were collected from the EMR: day of admission, for example, weekday or weekend, age, sex, WFNS grade, Fisher grade, surgical intervention (clip, coil, nonoperative) computed tomography (CT) imaging, commencement of enteral feeding, nursing patient at 30 degrees, stress ulcer prophylaxis, deep vein thrombosis (DVT) prophylaxis given (mechanical and pharmaceutical), maintenance of hemoglobin between 8 and $10 \mathrm{~g} / \mathrm{L}$, continuation of statin, and nimodipine administration. Hourly observations for mean arterial pressure (MAP), systolic blood pressure (SBP), and temperature were collected from admission to 72 hours after admission, length of stay, ICU outcome.

\section{Patient Eligibility}

The patient population was obtained from the ANZICS(Australia and New Zealand Intensive Care Society) Core Adult Database, categorized by Code 402 (Subarachnoid Hemorrhage, APACHE III-J nonoperative) or 1503 (Subarachnoid Hemorrhage, APACHE III-J postoperative), which is displayed in - Table 1. Inclusion criteria were that patients were correctly and formally diagnosed with an aSAH using digital subtraction angiography (DSA) and data for all domains were available from clinical records. Patients were excluded if they were admitted postendovascular coiling, or had a concurrent diagnosis of either intracerebral hemorrhage or subdural hemorrhage. This flowchart is displayed in - Fig. 1.

\section{Statistical Analysis}

Simple descriptives and frequencies were computed in SPSS Statistics Version 25.0 (IBM SPSS, Chicago, Illinois, United States). All significance tests were two-sided and a $p$-value

Table 1 Patients classified by ANZICS Codes 402 and Code 1503 between 2014 and 2016, denoted a formal diagnosis of "Subarachnoid Hemorrhage"

\begin{tabular}{|l|l|l|l|l|}
\hline & 2014 & 2015 & 2016 & $n$ (total) \\
\hline$n(402)$ & 22 & 15 & 15 & 52 \\
\hline$n(1,503)$ & 19 & 14 & 17 & 50 \\
\hline$n$ (total) & 41 & 29 & 32 & 102 \\
\hline
\end{tabular}

Abbreviation: ANZICS, Australia and New Zealand Intensive Care Society. 


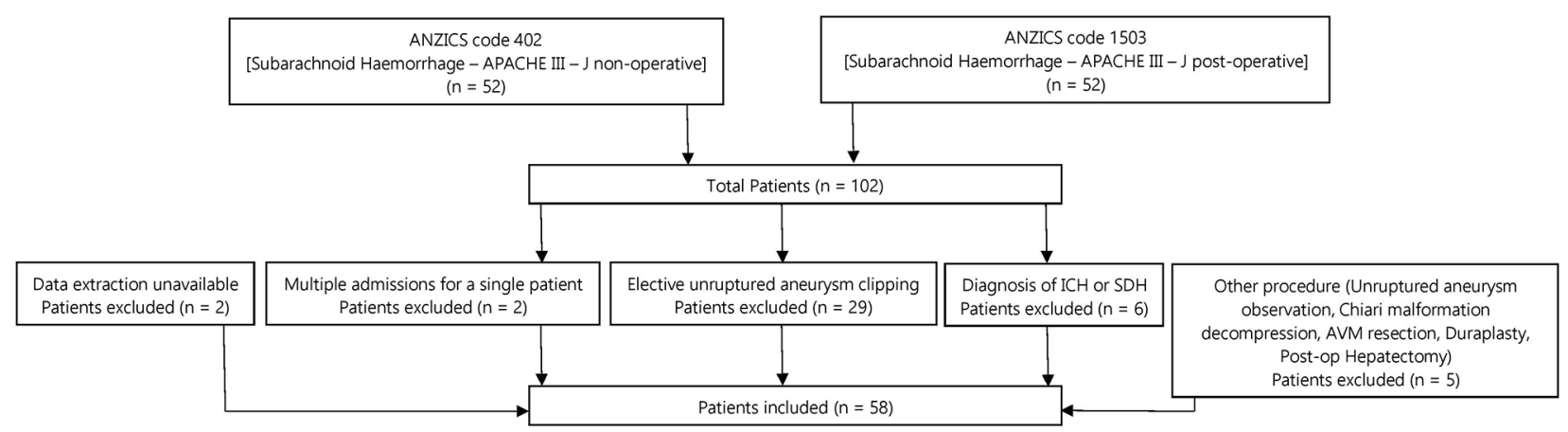

Fig. 1 Patient population before and after application of exclusion criteria. The most common reason for exclusion was due to patients being misdiagnosed with Australia and New Zealand Intensive Care Society (ANZICS) Codes that would otherwise suggest aneurysmal subarachnoid hemorrhage.

of 0.001 represented statistical significance. Simple twotailed nonparametric Kolmogorov-Smirnov tests were employed to determine normality of data. The median has been reported in addition to mean to provide a better measure of central tendency due to small patient population. Correlation statistics using Spearman's rho was used to measure the strength of association between two ranked variables: MAP, SBP, and temperature.

\section{Results}

\section{Patient Inclusion Assessment}

The eligibility flowchart ( - Fig. 1) shows 102 cases of aSAH were assessed for eligibility, 58 of which satisfied both inclusion and exclusion criteria. These patients formed the study population, and all patients who were diagnosed with aSAH on DSA were included in the study.

\section{Patient Characteristics}

Baseline characteristics of the study population are summarized in - Table 2 . The median patient age was 58.5 years old and 41 (70.7\%) patients were female, compared with $17(29.3 \%)$ males. In patients aged $>70$ years old, $13(93 \%)$ of these were female. The mean length of stay was $12.6( \pm 13.6)$ days, with $36(62.1 \%)$ patients being admitted on weekdays compared with $17(29.3 \%$ ) patients on weekends. All patients admitted underwent early CT imaging within the first 24 hours after admission. Of those patients, 9 (15.5\%) patients were declared braindead, 44 (75.9\%) discharged to another ward within the same hospital, and 5 (8.6\%) were transferred to a quaternary center with the intention of completing an endovascular coiling procedure, with all patients returning to the original ICU post-coiling. Therefore, $84.5 \%$ of admitted patients survived to ICU discharge. None of the patients were readmitted to the ICU, and ultimately discharged into the community, giving an in-hospital mortality rate of $15.5 \%$.

\section{aSAH Severity}

All patients should be classified according to the WFNS scoring system and Fisher scale. WFNS was classified and documented in $43(74.1 \%)$ patients during admission compared with 29 (50\%) who received a classified and documented Fisher grade. The radiologists, especially the interventional neuroradiologists, were involved, and they documented the Fisher scale in the CT report. However, the protocol requires it to be documented in the clinical notes.

There were $10(17.2 \%)$ patients who did not receive a WFNS or Fisher grade classification and documentation, and 7 (70\%) of these patients were discharged to the ward after nonsurgical conservative management. Of the 9 patients who developed brain-death, 6 (66\%) patients who were declared braindead had a median WFNS score of 5 . The remaining three (33\%) patients were not scored.

\section{Surgical Intervention}

A total of 42 (72.4\%) patients underwent surgery after diagnosis of aSAH. Of these patients, 37 patients (88\%) underwent clipping, and 5 (12\%) patients were transferred for coiling. The definitive management of the aneurysm was based upon its location and anatomy, and after multidisciplinary discussion between the neurosurgeon and interventional neuroradiologist. Of the 37 patients who underwent clipping, 31 (83.7\%) were within 48 hours of admission, and the remaining 6 patients (16.3\%) within 72 hours of admission.

Of the remaining 16 patients who did not underwent surgical intervention (27.6\% of all admissions), 9 (56\%) patients were managed conservatively (nonoperatively) due to the minor extent of the SAH, and 7 (46\%) patients were deemed to have a poor prognosis and further supportive care was not instituted. Surgical clipping was the intervention of choice, with 37 (88\%) patients having this procedure, and coiling was planned prospectively for 5 (12\%) patients, which required transfer to a quaternary center. Nonoperative measures were implemented in two subpopulations, those with minor bleeding and those with catastrophic bleedings who either developed brain-death or received palliative therapy.

\section{Supportive Care}

Early feeding was initiated within 72 hours in 45 (77.6\%) patients in the form of supervised oral nutrition (63.8\%) and nasogastric feeds (36.2\%). Positional nursing at 30 degrees was maintained in $54(93.1 \%)$ patients. 
Table 2 Patient factors, disease grade, and adherence to protocol

\begin{tabular}{|c|c|c|}
\hline \multicolumn{3}{|l|}{ Patient characteristics } \\
\hline \multirow[t]{3}{*}{ Age-y } & Mean ( \pm SD) & $56.4( \pm 15.5)$ \\
\hline & Median & 58.5 \\
\hline & Min, max & 16,88 \\
\hline \multirow[t]{2}{*}{ Sex-n (\%) } & Female & $41(70.7)$ \\
\hline & Male & $17(29.3)$ \\
\hline \multirow[t]{2}{*}{ Admission-n (\%) } & Weekday & $36(62.1)$ \\
\hline & Weekend & $22(37.9)$ \\
\hline \multirow[t]{3}{*}{ Length of stay-days } & Mean ( \pm SD) & $12.6( \pm 13.6)$ \\
\hline & Median & 8 \\
\hline & Min, max & 1,88 \\
\hline \multirow[t]{3}{*}{ Outcome-n (\%) } & Deceased & $9(15.5)$ \\
\hline & Discharged to ward & $44(75.9)$ \\
\hline & $\begin{array}{l}\text { Transferred to other } \\
\text { hospital }\end{array}$ & $5(8.6)$ \\
\hline CT performed-n (\%) & Yes & $58(100.0)$ \\
\hline$n=58$ & No & 0 \\
\hline Fisher grade-n (\%) & None & $29(50.0)$ \\
\hline \multirow[t]{4}{*}{$n=29$} & 1 & $3(5.2)$ \\
\hline & 2 & $2(3.5)$ \\
\hline & 3 & $6(10.3)$ \\
\hline & 4 & $18(31.0)$ \\
\hline WFNS score-n (\%) & None & $15(25.9)$ \\
\hline \multirow{5}{*}{$n=43$} & 1 & $19(32.8)$ \\
\hline & 2 & $6(10.3)$ \\
\hline & 3 & $1(1.7)$ \\
\hline & 4 & $5(8.6)$ \\
\hline & 5 & $12(20.7)$ \\
\hline Intervention type-n (\%) & Clip & $37(63.8)$ \\
\hline \multirow[t]{3}{*}{$n=58$} & Coil & $5(8.6)$ \\
\hline & Conservative & $9(15.5)$ \\
\hline & Futile & $7(12.1)$ \\
\hline Time to surgery-n (\%) & $>24 \mathrm{~h}$ & $10(23.8)$ \\
\hline \multirow[t]{2}{*}{$n=37$} & $24-48 \mathrm{~h}$ & $21(50.0)$ \\
\hline & $48-72 \mathrm{~h}$ & $6(14.3)$ \\
\hline Early feeding-n (\%) & Yes & $45(77.6)$ \\
\hline$n=58$ & No & $13(22.4)$ \\
\hline Nursed at 30 degrees $-n$ (\%) & Yes & $54(93.1)$ \\
\hline$n=58$ & No & $4(6.9)$ \\
\hline Stress ulcer prophylaxis-n (\%) & Yes & $54(93.1)$ \\
\hline$n=58$ & No & $4(6.9)$ \\
\hline Anemia prophylaxis-n (\%) & Yes & $9(100.0)$ \\
\hline$n=9$ & No & 0 \\
\hline DVT prophylaxis & Yes & $32(55)$ \\
\hline \multirow[t]{2}{*}{$n=58$} & No & 0 \\
\hline & Unknown & 26 \\
\hline Continue statins & Given & $6(66.7)$ \\
\hline$n=9$ & Not given & $3(33.3)$ \\
\hline Nimodipine & Administered & $49(84.5)$ \\
\hline \multirow[t]{2}{*}{$n=58$} & Not administered & $8(13.8)$ \\
\hline & Diltiazem given & $1(1.7)$ \\
\hline
\end{tabular}

Abbreviations: CT, computed tomography; DVT, deep vein thrombosis; SD, standard deviation; WFNS, World Federation of Neurosurgical Societies.

\section{Stress Ulcer Prophylaxis, pRBC Transfusion, and DVT Prophylaxis}

Within the patient population, 54 (94.1\%) patients received stress ulcer prophylaxis in the form of pantoprazole. All nine (15.5\%) patients with hemoglobin $<80 \mathrm{~g} / \mathrm{L}$ received packed red blood cells (pRBC). The anemic population unanimously had a recorded WFNS score of $>4$. Pneumatic mechanical calf-compressors were instituted in all patients; however, pharmaceutical DVT prophylaxis was given in 32 (55\%) eligible patients, with 26 patients having missing data for this outcome. Even with missing data this is a low figure and raises concerns since this decision often leads to disagreements between neurosurgical and intensive care teams. The lack of documentation may reflect a lack of discussion of what is often an area of disagreement in management.

\section{Statins}

Prior to admission, nine (15.5\%) patients were taking a statin as regular medication, of those patients, 6 (66.7\%) continued to receive during their admission.

\section{Vasospasm and Nimodipine}

Vasospasm management included either intravenous or oral nimodipine for 21 days, along with an appropriate fluid and blood pressure management, as per the protocol. Further vasospasm management involved as cerebral intra-arterial verapamil injections, if deemed necessary at subsequent DSA. All other complications were managed as per the treating intensivist. Of the total subjects, 49 (84.5\%) patients were given nimodipine, while eight (13.8\%) patients did not receive a calcium channel blocker. Diltiazem was given as an alternative to nimodipine in one patient. This was clinician choice, with no clear explanation, although the patient was known to have atrial fibrillation.

\section{Observations}

Total numbers of hourly observations and percentage of possible observations taken are summarized in - Table 3.

\section{Systolic Blood Pressure}

Of a possible 4,176 observations, 2,856 (68.3\%) were recorded for 58 patients in the first 72 hours after admission. The mean SBP was $133.8 \mathrm{~mm} \mathrm{Hg}$ (standard deviation [SD] $= \pm 21.8$, median $=132$ ), with a range of $156 \mathrm{~mm} \mathrm{Hg}$ (min: 65, max: 221 ). About $11.5 \%$ (329) of SBP readings taken were $>160$ $\mathrm{mm} \mathrm{Hg}$, outside of protocol range. Patients had a mean of $82.4 \%$ ( $S D= \pm 0.59$, median $=85$ ) recordings taken within protocol requirements in the first 72 hours after admission.

\section{Mean Arterial Pressure}

Of a possible 4,176 observations, a total of 2,815 (67.4\%) MAP observations were recorded, with a MAP of $86.3 \mathrm{~mm}$ $\mathrm{Hg}(\mathrm{SD}= \pm 12.8$, median $=132)$ and range of $94 \mathrm{~mm} \mathrm{Hg}$ (min: 45 , max: 139). About $4.4 \%$ (123) of MAP readings taken were outside the recommended range of 65 to $110 \mathrm{~mm} \mathrm{Hg}$.

Patients had a mean of $82.1 \%(\mathrm{SD}= \pm 0.13$, median $=82.5$ ) recordings taken within protocol requirements in the first 72 hours after admission. 


\section{Temperature}

A total of $1,679(40.2 \%)$ temperature observations were recorded, with a mean temperature of $37.1^{\circ} \mathrm{C}$. $(\mathrm{SD}= \pm 0.59$, median: 37.1) and range of $5.2^{\circ} \mathrm{C}(\min : 33.8, \max : 39)$. Thirty-two percent (538) of temperature readings taken were outside of the normothermic range of 36.5 to $37.5^{\circ} \mathrm{C}$. Patients recordings had a mean adherence of $47.5 \%$ $(\mathrm{SD}= \pm 0.24$, median $=40)$.

\section{Relationships: Demographics, Observations, and Injury Severity}

Factors of age and sex do not have a relationship with how regularly SBP, MAP, or temperature recordings are taken in the first 72 hours after admission, nor does admission to ICU on weekends versus weekdays. There is a significant correlation $\left(r_{s}=0.998, p<0.001\right)$ between how often SBP and MAP are measured, suggesting that as the number of SBP readings documented increases as do the number of MAP readings. The protocol states that MAP is to be the observed and monitored blood pressure parameter; however, there are often requests for a specific SBP to be maintained at the same time. The increasing number of SBP measurements in response to MAP measurements likely represents discrepancies between the two desired parameters, and the human-factor needs to have at least one parameter within limits. It is unlikely to represent damping interference with the arterial catheter measuring the MAP, as the MAP would stay the same; however, it may indicate that the arterial catheter was no longer reliable, leading to a greater reliance on the SBP. Increase in WFNS score is associated with an increase in Fisher grade $\left(r_{s}=0.857, p<0.001\right)$.

\section{Discussion}

This study has provided insight into the diagnosis and management of the aSAH patient population admitted to our ICU between 2014 and 2016. Demographic data collected from this study accurately reflects the known epidemiology of aSAH in Australia. ${ }^{22,23}$ The mean age of presentation in this study was 56.4 years, which closely mirrors the Australasian Cooperative Research on Subarachnoid Hemorrhage Study findings of 57 years, ${ }^{23}$ with female sex and increasing age as established risk factors. The peak age group for patients to experience aSAH was between 45 and 64 years, accounting for almost $48 \%$ of admissions to ICU. The Macquarie study found a similar peak in admissions for this age group at $45 \%$ during 1998 to $2008 .{ }^{22}$ Comparison of the results of this study to the current literature surrounding epidemiology across Australia identified that despite a small cohort size, the demographics from this study are consistent with the trends seen in other aSAH populations.

This study highlights multiple areas of strength and weakness regarding protocol adherence. CT was diligently performed prior to admission to ICU in all patients, which is essential for clarification of clinical diagnosis. About $73.8 \%$ of patients who were eligible for surgical clipping at Nepean received prompt management within 48 hours of admission.
Table 3 Total numbers of hourly observations and percentage of possible observations taken

\begin{tabular}{|c|c|c|}
\hline \multicolumn{3}{|l|}{ Hourly observations } \\
\hline $\mathrm{SBP}(\mathrm{mm} \mathrm{Hg})$ & Mean $( \pm$ SD) & $133.8( \pm 21.8)$ \\
\hline \multirow[t]{2}{*}{$n=2,856$} & Median & 132 \\
\hline & Min, $\max$ & 65,221 \\
\hline $\operatorname{MAP}(\mathrm{mm} \mathrm{Hg})$ & Mean $( \pm S D)$ & $86.3( \pm 12.8)$ \\
\hline \multirow[t]{2}{*}{$n=2,815$} & Median & 85 \\
\hline & Mix, max & 45,139 \\
\hline Temperature $\left({ }^{\circ} \mathrm{C}\right)$ & Mean $( \pm$ SD) & $37.1( \pm 0.59)$ \\
\hline \multirow[t]{2}{*}{$n=1,679$} & Median & 37.1 \\
\hline & Min, $\max$ & $33.8,39$ \\
\hline SBP adherence- $n$ (\%) & Mean $( \pm S D)$ & $82.4( \pm 0.13)$ \\
\hline \multirow[t]{2}{*}{$n=58$} & Median & 82.5 \\
\hline & Min, $\max$ & 49,100 \\
\hline MAP adherence-n (\%) & Mean $( \pm$ SD) & $82.1( \pm 0.13)$ \\
\hline \multirow[t]{2}{*}{$n=58$} & Median & 82.5 \\
\hline & Min, max & 49,100 \\
\hline $\begin{array}{l}\text { Temperature } \\
\text { adherence-n (\%) }\end{array}$ & Mean $( \pm S D)$ & $47.5( \pm 0.24)$ \\
\hline \multirow[t]{2}{*}{$n=58$} & Median & 40 \\
\hline & Min, max & 0,100 \\
\hline
\end{tabular}

Abbreviations: MAP, mean arterial pressure; SBP, systolic blood pressure; SD, standard deviation.

The patient population had $93.1 \%$ adherence for achieving positional venous drainage and stress ulcer prophylaxis in the form of pantoprazole. Additionally, 49 (82.6\%) of patients received calcium channel blockers in the form of nimodipine, and in one case diltiazem. Of the nine patients with a hemoglobin $<80 \mathrm{~g} / \mathrm{L}, 100 \%$ received $\mathrm{pRBC}$. SBP and MAP were recorded within the protocol recommendations $(<160$ and $65-110 \mathrm{~mm} \mathrm{Hg}$ ) with adherences of 82.4 and $82.1 \%$, respectively, during the first 72 hours after admission.

Protocol adherence for hourly SBP, MAP, and temperature recordings is independent of demographic factors including sex and age, day of admission, WFNS score, and admission outcome. This suggests that regardless of these factors, all patients receive the same high level of care.

A weakness in the study finding was surrounding aSAH scoring systems. The "management of aSAH protocol" requires the use of one of two classification systems based on clinical examination on presentation: the Hunt and Hess score and the WFNS score. This study clearly demonstrated that the WFNS score was the preferred system, as no patients received a Hunt and Hess classification. ${ }^{24,25}$ The WFNS score is based broadly on Glasgow Coma Scale combined with the presence of absence of focal neurological deficits, ${ }^{26}$ and is likely preferred due to its immediate clinical applicability and ease of implementation. In addition to clinical scoring, Fisher scale was noted in only $50 \%$ of patients, despite all patients receiving early CT imaging. This highlights a potential missed opportunity for radiological interpretation and vasospasm-risk classification. ${ }^{27}$ 
Correlation studies showed no association between outcome at 22 days after admission based on WFNS or Fisher classification. This poses questions as to the necessity for classification systems to be included in the protocol. Studies have shown that almost $50 \%$ of patients with poor WFNS scores at admission have good outcomes, ${ }^{28}$ and some studies suggest that it is currently not beneficial to strongly advocate universal adoption of any aSAH grading scales..$^{29}$ Looking forward, this suggests the need for a novel or modified grading scale that encompasses both physical impairment and vasospasm risk.

Pharmaceutical DVT prophylaxis for the first 72 hours of admission was poorly documented within patient records. Of the 58 patients, 32 (55\%) received 5,000 units of subcutaneous heparin, twice daily. In the remaining 26 (45\%) patients, it was unknown as to whether they were ineligible, did not receive prophylaxis, or had calf compressors applied as a nonpharmaceutical alternative. Of the nine patients taking statins prior to admission, six (66.7\%) patients had continuity of administration. Notably the Simvastatin in Aneurysmal Subarachnoid Hemorrhage trial recently showed that there was no attributable effect of continuing a prior dose of simvastatin on clinical outcome after aSAH. ${ }^{30,31}$

Temperature was a poorly recorded observation in comparison to SBP and MAP. The mean adherence was $47.5 \%$ within protocol recommendations $\left(36.5-37.5^{\circ} \mathrm{C}\right)$, with $22.8 \%$ (538) of individual observations occurring outside of the recommended range. Thirty-two percent (382) of observations were above $37.5^{\circ} \mathrm{C}$ and $3 \%$ (51) of these fell into the neurogenic fever range, which is well characterized complication of aSAH. ${ }^{32-35}$

This study has substantial strength in that the methods devised for data collection are innovative, tailored, and are applicable to future protocol quality analysis in our ICU. This study is a novel venture and has set the scene for further analysis in the management of aSAH, with the intention of refining future methods to mitigate bias. In addition, this study has identified incidences of suboptimal use of the ANZICS Database codes. Improvement in ICU diagnostic classifications will further assist with data collection and prospective research.

A further strength of this study is that literature states that the modifiable factors for SAH management are preoperative rebleeding and $\mathrm{DCI}$. These potential complications benefit best from early surgical management and strict BP control. It is adherence to protocols that enable these parameters to be met, thus ensuring good outcomes. This study shows good adherence to the most impactful areas of aSAH management, which focuses the further discussion on ensuring the other areas of management, as well as improving communication as to why protocols are being deviated from. ${ }^{36}$ We have also demonstrated that our in-hospital outcomes are favorable compared with international outcomes, with our in-hospital mortality being $15.5 \%,{ }^{37,38}$ compared with international figures being between 20 and 50\%, and the in-hospital mortality from the Columbia University SAH Outcomes Project being $18 \%{ }^{6}$
The limitations to this study are a relatively small sample size and some missing data points. Patient eligibility assessment excluded $43 \%$ of the initial sample population, due to incorrect ANZICS classification codes ( - Fig. 1). Patients were only followed up until 22 days post-aSAH, without exploring data on long-term outcomes. The lack of quantifiable data available permitted only a rudimentary understanding of adherence and the relationship between patient and disease factors. Further studies using a mixed-method design would be able to explore and illuminate the reasons why clinicians, both junior and senior deviated from the protocol. While the protocol states that reasons must be stated for any deviations from the protocol, the commonest entry in the clinical notes was "consultant decision." This is a significant human-factors issue, in that a data entry of this type gives no clinical reasoning for protocol deviation; it merely states who decided it. This is not helpful to other team members when they are trying to make subsequent management decisions that require the vital clinical reasoning for protocol deviation. Given the retrospective nature of this study, selection bias is a concern, as patients were not randomized. Measurement bias is a significant issue as there was no way to ensure data collected was originally reported with accuracy. In addition, inclusive bias remains an issue as only select segments of the protocol were assessed due to feasibility of time-sensitive data collection. Finally, this research was undertaken within a single center, restricting our insight of accurate case numbers of aSAH. The applicability of this data to other centers is limited as the focus is within a single department.

\section{Conclusion}

This study has explored adherence to our ICU protocol in the management of aSAH in adults. This study demonstrated that there was no association between variation in protocol adherence based on age, admission dates, or disease factors including Fisher and WFNS scores, suggesting a culture for treating all patients according to protocol, and not based on any subjective bias. The current management of aSAH within our ICU has strengths, primarily in early CT imaging, surgical intervention, and blood pressure control, which are the areas that will have greatest impact on overall outcome. Areas for improvement were documentation of the correct classification of the aSAH using WFNS and Fisher grading, and management and accurate documentation of temperature measurement.

\section{Conflict of Interest}

None declared.

\section{References}

1 Udy AA, Vladic C, Saxby ER, et al. Australian and New Zealand Intensive Care Society Centre for Outcome and Resource Evaluation. Subarachnoid hemorrhage patients admitted to intensive care in Australia and New Zealand: a multicenter 
cohort analysis of in-hospital mortality over 15 years. Crit Care Med 2017;45(2):e138-e145

2 Rivero Rodríguez D, Scherle Matamoros C, Cúe LF, Miranda Hernández JL, Pernas Sánchez Y, Pérez Nellar J. Predictor's of mortality in patients with aneurysmal subarachnoid haemorrhage and rebleding. Neurol Res Int 2015;2015:545407

3 Zacharia BE, Hickman ZL, Grobelny BT, et al. Epidemiology of aneurysmal subarachnoid hemorrhage. Neurosurg Clin $\mathrm{N}$ Am 2010;21(2):221-233

4 van Gijn J, Rinkel GJE. Subarachnoid haemorrhage: diagnosis, causes and management. Brain 2001;124(Pt 2):249-278

5 Cesarini KG, Hårdemark $\mathrm{HG}$, Persson L. Improved survival after aneurysmal subarachnoid hemorrhage: review of case management during a 12-year period. J Neurosurg 1999;90(4):664-672

6 Lantigua H, Ortega-Gutierrez S, Schmidt JM, et al. Subarachnoid hemorrhage: who dies, and why? Crit Care 2015;19(1):309-309

7 Naidech AM, Janjua N, Kreiter KT, et al. Predictors and impact of aneurysm rebleeding after subarachnoid hemorrhage. Arch Neurol 2005;62(3):410-416

8 Connolly ES Jr, Rabinstein AA, Carhuapoma JR, et al; American Heart Association Stroke Council, Council on Cardiovascular Radiology and Intervention, Council on Cardiovascular Nursing, Council on Cardiovascular Surgery and Anesthesia, Council on Clinical Cardiology. Guidelines for the management of aneurysmal subarachnoid hemorrhage: a guideline for healthcare professionals from the American Heart Association/American Stroke Association. Stroke 2012;43(6):1711-1737

9 Steiner $T$, Juvela $S$, Unterberg $A$, Jung $C$, Forsting $M$, Rinkel G; European Stroke Organization. European Stroke Organization guidelines for the management of intracranial aneurysms and subarachnoid haemorrhage. Cerebrovasc Dis 2013;35(2):93-112

10 Dewan MC, Mocco J. Current practice regarding seizure prophylaxis in aneurysmal subarachnoid hemorrhage across academic centers. J Neurointerv Surg 2015;7(2):146-149

11 Rhoney DH, Tipps LB, Murry KR, Basham MC, Michael DB, Coplin WM. Anticonvulsant prophylaxis and timing of seizures after aneurysmal subarachnoid hemorrhage. Neurology 2000;55(2):258-265

12 Roos Y; STAR Study Group. Antifibrinolytic treatment in subarachnoid hemorrhage: a randomized placebo-controlled trial. Neurology 2000;54(1):77-82

13 McDougall CG, Spetzler RF, Zabramski JM, et al. The Barrow Ruptured Aneurysm Trial. J Neurosurg 2012;116(1):135-144

14 Molyneux AJ, Kerr RS, Yu LM, et al; International Subarachnoid Aneurysm Trial (ISAT) Collaborative Group. International Subarachnoid Aneurysm Trial (ISAT) of neurosurgical clipping versus endovascular coiling in 2143 patients with ruptured intracranial aneurysms: a randomised comparison of effects on survival, dependency, seizures, rebleeding, subgroups, and aneurysm occlusion. Lancet 2005;366(9488):809-817

15 Molyneux AJ, Birks J, Clarke A, Sneade M, Kerr RS. The durability of endovascular coiling versus neurosurgical clipping of ruptured cerebral aneurysms: 18 year follow-up of the UK cohort of the International Subarachnoid Aneurysm Trial (ISAT) Lancet 2015;385(9969):691-697

16 Worthington JM, Goumas C, Jalaludin B, Gattellari M. Decreasing risk of fatal subarachnoid hemorrhage and other epidemiological trends in the era of coiling implementation in Australia. Front Neurol 2017;8(424):424

17 Morris AH. Rational use of computerized protocols in the intensive care unit. Crit Care 2001;5(5):249-254

18 Wall RJ, Dittus RS, Ely EW. Protocol-driven care in the intensive care unit: a tool for quality. Crit Care 2001;5(6):283-285
19 Woolf SH, Grol R, Hutchinson A, Eccles M, Grimshaw J. Clinical guidelines: potential benefits, limitations, and harms of clinical guidelines. BMJ 1999;318(7182):527-530

20 Shekelle P, Eccles MP, Grimshaw JM, Woolf SH. When should clinical guidelines be updated? BMJ 2001;323(7305):155-157

21 Vernooij RWM, Sanabria AJ, Solà I, Alonso-Coello P, Martínez García L. Guidance for updating clinical practice guidelines: a systematic review of methodological handbooks. Implement Sci 2014;9:3-4

22 Lai L, Morgan MK. Incidence of subarachnoid haemorrhage: an Australian national hospital morbidity database analysis. J Clin Neurosci 2012;19(5):733-739

23 Epidemiology of aneurysmal subarachnoid hemorrhage in Australia and New Zealand: incidence and case fatality from the Australasian Cooperative Research on Subarachnoid Hemorrhage Study (ACROSS) Stroke 2000;31(8):1843-1850

24 Cedzich C, Roth A. Neurological and psychosocial outcome after subarachnoid haemorrhage, and the hunt and hess scale as a predictor of clinical outcome. Zentralbl Neurochir 2005;66(3):112-118

25 Hunt WE, Hess RM. Surgical risk as related to time of intervention in the repair of intracranial aneurysms. J Neurosurg 1968;28(1):14-20

26 Report of World Federation of Neurological Surgeons Committee on a Universal Subarachnoid Hemorrhage Grading Scale. J Neurosurg 1988;68(6):985-986

27 Fisher CM, Kistler JP, Davis JM. Relation of cerebral vasospasm to subarachnoid hemorrhage visualized by computerized tomographic scanning. Neurosurgery 1980;6(1):1-9

28 Taylor CJ, Robertson F, Brealey D, et al. Outcome in poor grade subarachnoid hemorrhage patients treated with acute endovascular coiling of aneurysms and aggressive intensive care. Neurocrit Care 2011;14(3):341-347

29 Rosen DS, Macdonald RL. Subarachnoid hemorrhage grading scales: a systematic review. Neurocrit Care 2005;2(2):110-118

30 Kirkpatrick PJ, Turner CL, Smith C, Hutchinson PJ, Murray GD; STASH Collaborators. Simvastatin in Aneurysmal Subarachnoid Haemorrhage (STASH): a multicentre randomised phase 3 trial. Lancet Neurol 2014;13(7):666-675

31 Su SH, Xu W, Hai J, Wu YF, Yu F. Effects of statins-use for patients with aneurysmal subarachnoid hemorrhage: a meta-analysis of randomized controlled trials. Sci Rep 2014;4:4573

32 Hocker SE, Tian L, Li G, Steckelberg JM, Mandrekar JN, Rabinstein AA. Indicators of central fever in the neurologic intensive care unit. JAMA Neurol 2013;70(12):1499-1504

33 Honig A, Michael S, Eliahou R, Leker RR. Central fever in patients with spontaneous intracerebral hemorrhage: predicting factors and impact on outcome. BMC Neurol 2015;15:6

34 Rabinstein AA, Sandhu K. Non-infectious fever in the neurological intensive care unit: incidence, causes and predictors. J Neurol Neurosurg Psychiatry 2007;78(11):1278-1280

35 Thompson HJ, Pinto-Martin J, Bullock MR. Neurogenic fever after traumatic brain injury: an epidemiological study. J Neurol Neurosurg Psychiatry 2003;74(5):614-619

36 Galea JP, Dulhanty L, Patel HC; UK and Ireland Subarachnoid Hemorrhage Database Collaborators. Predictors of outcome in aneurysmal subarachnoid hemorrhage patients: observations from a multicenter data set. Stroke 2017;48(11):2958-2963

37 Longstreth WT Jr, Nelson LM, Koepsell TD, van Belle G. Clinical course of spontaneous subarachnoid hemorrhage: a population-based study in King County, Washington. Neurology 1993;43(4):712-718

38 Sudlow CL, Warlow CP; International Stroke Incidence Collaboration. Comparable studies of the incidence of stroke and its pathological types: results from an international collaboration. Stroke 1997;28(3):491-499 